\title{
Uncertainty levels are determined based on experimental data
}

\author{
Fu-Cun Qu \\ Chengdu Information Engineering University College of Control Engineering, Chengdu 610225, \\ Sichuan Province, China \\ qufc@cuit.edu.cn
}

Key words: Robust control,Uncertainty, Model identification

Abstract: In the robust control theory, the norm boundary of uncertainty set (i.e. the uncertainty level) is an important issue. When the level of uncertainty is too large, the results will be too conservative, and when the uncertainty is too small, the results will be too optimistic. Two methods for determining the uncertainty level based on experimental data are given in this paper. Method 1 can obtain the minimum model error bounds, but the calculation is complex. Method 2 is simple in calculation and suitable for engineering applications.

\section{Introduction}

In the theory of robust control, Control system was seen as a family of systems, Can be formed by a nominal system and a perturbation, perturbation taken using a norm bounded set $\Delta$. So how to reasonably determine the collection $\Delta$ level of uncertainty is an important problem. Because when uncertainties beyond the actual model error, mu analysis method will be too conservative, and when uncertainty is lower than the actual model error, mu analysis method will be overly optimistic conclusion.

\section{Minimum model set method}

Determine whether a model uncertainty level with satisfaction it is difficult to reflect the real dynamic characteristics of the object. Because the real dynamic characteristics of the object in the general case is unknowable. Rely solely on under test signal excitation input and output signal of the measured values, and some according to its physical properties or other experimental data to get a priori information, often can't completely decided to nominal model and the real dynamic characteristics of the differences between control object.

However, if a control object model and the incompatibility between the information, then the model do not have the ability to describe the real dynamic characteristics of controlled object. Therefore, in essence, to determine the collection $\Delta$ level of uncertainty is a unfalsification process, rather than a verfication process.

In all of the nominal model, which has the minimum error bounds? The problem in a robust identification theory known as most powerful unfalsified modeling. It follows the principle is: model should not contradict with the experimental data and has the most "simple" structure.

For single input single output system, and with additive disturbances, the most powerful unfalsified modeling, have the following theorem:

Theorem: A control object ture transfer function $\mathrm{G}(\lambda)$ can be represented as: $G(\lambda)=G_{0}(\lambda)+\Delta(\lambda) W(\lambda)$ 
$W(\lambda) \in H_{\infty}, \Delta(\lambda) \in H_{\infty},\|\Delta(\lambda)\|_{\infty} \leq 1, G_{0}(\lambda)=\sum_{i=0}^{p} a_{i} \lambda^{i}\left(1+\sum_{i=0}^{p} b_{i} \lambda^{i}\right)^{-1}, \mathrm{~b}_{i}$ for known constant.

Given a transfer function with minimum phase characteristics: $W_{0}(\lambda)=\sum_{i=0}^{\mathrm{q}} c_{i} \lambda^{i}\left(1+\sum_{i=0}^{q} \mathrm{~d}_{i} \lambda^{i}\right)^{-1}$

And group m time-domain input and output experimental data, the control object's initial state is zero: $\left.\left(\mathrm{u}_{k}(\mathrm{i}), y_{k}(\mathrm{i})\right)\right|_{k=0} ^{N-1}, i=1,2, \mathrm{~L}, m$ 。

$\Phi$ model set is defined as: $\Phi=\left\{\mathrm{G}(\lambda) \mid \mathrm{G}(\lambda)=\mathrm{G}_{0}(\lambda)+\Delta(\lambda) W(\lambda),\|\Delta(\lambda)\|_{\infty} \leq 1\right\}$

All the experimental data can be regeneration model set $\Phi$, and to minimize the objective function. If and only if: $\mathrm{W}(\lambda)=\beta W_{0}^{-1}(\lambda)$

$$
\begin{aligned}
& \beta=\max _{1 \leq i \leq m} \bar{\sigma}\left\{\left[T_{z}(u(i))-T_{z}\left(w_{u}(i)\right)\right]^{-1} T_{z}^{-1}(d(i)) T_{z}^{-1}(b(i)) T_{z}(c(i)) \times\right. \\
& {\left[T_{z}(y(i))-T_{z}\left(w_{y}(i)\right) T_{z}(b(i))-\left(T_{z}(u(i))-T_{z}\left(w_{u}(i)\right) T_{z}(a(i))\right]\right\}}
\end{aligned}
$$

$\mathrm{Tz}(*)$ is a lower triangular toeplits matrix.

See from the above theorem, calculating minimum model that involves the optimization problem of matrix eigenvalues, the calculation is very complicated, is not convenient for engineering application.

\section{Model effectiveness method}

Model effectiveness's algorithm can simplify the above algorithm, Although it is not the smallest model sets, but you can get a reasonable level of uncertainty.

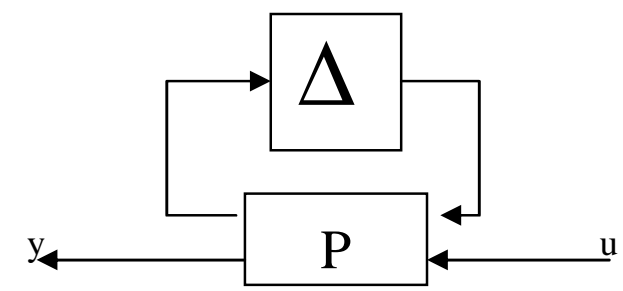

Figurea 1. Structure diagram of system model

Definition of uncertainty effectiveness: As shown in Figure 1, the existence of a $\Delta \in \Delta$ set makes $F_{u}(P, \Delta)$ possible to produce the measured $\mathrm{y}$ and $\mathrm{u}$, If so, the system with uncertainty $\Delta$ is effectiveness, otherwise it is invalid.

The effectiveness of the model and the minimum model set identification problem are essentially different, it does not find the minimal model set, but only to determine whether there is effectively uncertainty $\Delta \in \Delta$.

According to the robust stability theorem, for the robust stable system, the input and output experimental data are given $\left(\mathrm{u}_{\mathrm{i}}, y_{i}\right)_{\mathrm{i}=1}^{n}$, When the following conditions are true, the systems with 
uncertainty $\Delta \in \Delta$ is effective: $\left.\mu_{\Delta} \mid P_{11}-\hat{P}_{12} \hat{P}_{22}^{-1} P_{21}\right\rfloor>1$, Among them, $P=\left[\begin{array}{ll}P_{11} & P_{12} \\ P_{21} & P_{22}\end{array}\right]$ $\hat{P}_{12}=P_{12} \mathrm{u}, \quad \hat{P}_{22}=P_{22} \mathrm{u}-y$

This condition seems contrary to the intuitive, because the effectiveness condition is given by $\mu>$ 1 , rather than robust stability condition of the $\mu<1$.

This can be illustrated by following formula: $\mathrm{y}=F_{u}(P, \Delta) u$

$\left[P_{22} \mathrm{u}-y\right]+P_{21} \Delta\left(I-P_{11} \Delta\right)^{-1}\left[P_{12} u\right]=\hat{P}_{22}+P_{21} \Delta\left(I-P_{11} \Delta\right)^{-1} \hat{P}_{12}=0$

Definition $\hat{P}=\left[\begin{array}{ll}P_{11} & \hat{P}_{12} \\ P_{21} & \hat{P}_{22}\end{array}\right]$, When $\mu_{\Delta}(\hat{P})<1$, it is shown that the system with the uncertainty $\Delta$ is robust stable, And show that $F_{u}(\hat{P}, \Delta)$ is nonsingular. To meet $\mu_{\Delta}(\hat{P})<1$ would be inconsistent with the above equation, This equation requires $F_{u}(\hat{P}, \Delta)$ is singular, so as to satisfy the relationship between input and output data.

The size of $\mu$ can be used as a measure rationality of uncertainty $\Delta$. For example, $\mu=2$ indicates the level of uncertainty can be reduced by half, $\mu=0.5$ indicates uncertainty $\Delta$ invalid, need to increase twice. Based on this principle, an algorithm for calculating the level of reasonable uncertainty using experimental data is given.

Algorithm for determining uncertainty levels: Given experimental data(u(i), $y(\mathrm{i}))_{\mathrm{i}=1}^{n}$, Given system nominal model $P=\left[\begin{array}{ll}P_{11} & P_{12} \\ P_{21} & P_{22}\end{array}\right]$, Given uncertainty set $\Delta$ and weight coefficient $\mathrm{W}$, the model set $\Phi$ is: $\Phi=\left\{\mathrm{F}_{\mathrm{u}}(P, W \Delta),\|\Delta\|_{\infty} \leq 1\right\}$, Given a reasonable small quantity $\varepsilon>0$

1.Read the first i(u(i), $y(\mathrm{i}))$

2.Calculate $\hat{P}_{12}=P_{12} \mathrm{u}_{\mathrm{i}}, \quad \hat{P}_{22}=P_{22} \mathrm{u}_{\mathrm{i}}-y_{i}$

3. Calculate $\beta=\mu_{\Delta}\left[P_{11}-\hat{P}_{12} \hat{P}_{22}^{-1} P_{21}\right\rfloor$

4. if $\beta<1$ or $\beta>1+\varepsilon$, then $P_{11}=P_{11} / \beta, \mathrm{W}=\mathrm{W} / \beta$, turning step 3

5.if $1<\beta<1+\varepsilon$, through the model effectiveness check, $\mathrm{i}=\mathrm{i}+1$, turning step 1 $\mathrm{W}$ represents the level of uncertainty of the system here.

\section{Summary}

In the application of robust control theory, it is very important to reasonably determine the norm boundary of uncertainty set $\Delta$ (i.e. uncertainty level), which is one of the major factors determining whether the application is successful or not.

In essence, determining the uncertainty level of set $\Delta$ is a "inspection false" process, not a "verfication" process. 
The modeling methods are different for different purposes and, naturally, model checking methods are also different. The size of the uncertainty level is related to model checking methods and standards.

For a system with single input, single output and additive disturbance, the method of calculating the minimum uncertainty level is given. This method is difficult to be used in engineering because of its complex calculation.

Based on the principle of model effectiveness is given the algorithms to use experimental data to calculate a reasonable level of uncertainty. The method is simple and convenient for engineering applications.

\section{References}

[1] $\mathrm{Qu}$ fucun, shi zhongke. Application of robust flutter margin method. Flight dynamics. VOL.24,No.1(2006)70-72.

[2] Zhou tong. Introduction to control-oriented system identification. Tsinghua University press, Beijing, 2004.

[3] Rick Lind, Martin J. Brenner. Robust Flutter Margin Analysis That Incorporates Flight Data. NASA/TP-1998-206543. 